\title{
Corotating and irrotational binary black holes in quasi-circular orbits
}

\author{
Gregory B. Cook \\ Department of Physics, Wake Forest University, Winston-Salem, North Carolina 27109
}

(Dated: November 16, 2018)

\begin{abstract}
A complete formalism for constructing initial data representing black-hole binaries in quasiequilibrium is developed. Radiation reaction prohibits, in general, true equilibrium binary configurations. However, when the timescale for orbital decay is much longer than the orbital period, a binary can be considered to be in quasi-equilibrium. If each black hole is assumed to be in quasi-equilibrium, then a complete set of boundary conditions for all initial data variables can be developed. These boundary conditions are applied on the apparent horizon of each black hole, and in fact force a specified surface to be an apparent horizon. A global assumption of quasi-equilibrium is also used to fix some of the freely specifiable pieces of the initial data and to uniquely fix the asymptotic boundary conditions. This formalism should allow for the construction of completely general quasi-equilibrium black hole binary initial data.
\end{abstract}

PACS numbers: 04.20.-q, 04.25.Dm, 04.70.Bw, 97.80.-d

\section{INTRODUCTION}

The problem of calculating the gravitational waveforms produced during the final plunge and coalescence of a pair of black holes currently is receiving considerable attention. This attention is motivated by the imminent deployment of the Laser Interferometric Gravitational Wave Observatory (LIGO) and other, similar, detectors. It is further motivated by the fact that blackhole binaries are expected to be strong sources of gravitational waves and are considered one of the most likely candidates for early detection. The detection and interpretation of black hole mergers will be greatly facilitated by theoretical predictions for the gravitational waveforms produced by these events. However, in the absence of predictions for the full waveforms, even information about the behavior of the orbits will be helpful. In particular, information about the properties of the so-called last stable orbit, or innermost stable circular orbit (ISCO), is of interest.

Currently, large scale numerical simulations are the only known approach for computing the gravitational waveforms produced during the final plunge and coalescence of black-hole binaries. Any dynamical simulation must start with some initial data, and the results of such a simulation are entirely dependent on how well the given initial data correspond to the desired physical situation. Thus, astrophysically realistic initial data are required if we are going to simulate the gravitational waveforms of astrophysical black-hole binaries. Because radiation damping tends to circularize binary orbits, orbits that are nearly circular are of greatest interest. Of course, energy loss by gravitational radiation also means that no truly circular orbits can exist for black-hole binary systems. To keep this fact in mind, we will always refer to such orbits as being quasi-circular.

*Electronic address: cookgb@wfu.edu
The usefulness of current numerical simulations of general black-hole binary systems is limited by two important facts. First, the simulations themselves are only beginning to reach the stage where they can produce useful information. Furthermore, the accuracy of this information is still questionable. Second, the initial data currently being used for these simulations is rather crude. Initial data for binary black hole systems have been available for some time now [1, 2]. The approaches for generating these initial data can produce rather arbitrary configurations and a way of picking which data sets represent binaries in quasi-circular orbits was needed. An approximation scheme for locating quasi-circular orbits was developed[3]. Based on an effective-potential method, this scheme was first applied to non-spinning, equal-mass binaries using the inversion-symmetric data of Ref. [1]. Later, this approach was applied to non-spinning, equalmass binaries based on puncture data [4] and to spinning, equal-mass black holes $[5]$.

Unfortunately, there are problems with these data sets. Because the schemes used to generate this data assume the metric to be conformally flat, they cannot produce astrophysically accurate data for spinning holes 5 . Furthermore, the estimates for the location of the ISCO produced by these schemes do not agree well with results based on various post-Newtonian approximation methods [6, 7]. There is some speculation that this discrepancy is rooted in the approximation of conformal flatness. While the conformal flatness approximation certainly introduces some error, I suspect that the most important source of error comes from a failure to accurately approximate the masses of the black holes as they approach the ISCO.

Initial-data schemes relying on the conformal flatness approximation have been popular because they are relatively simple and inexpensive from a computational point of view. When maximal slicing is used, an analytic solution of the momentum constraints exists [8, 9]. This means that only the Hamiltonian constraint, a single quasi-linear elliptic PDE, need be solved. Recently, ef- 
forts have been made to move beyond the approximation of conformal flatness (cf. Ref. 10, 11). Unfortunately, these data have not yet been used to estimate the location of the ISCO.

For the case of neutron-star binaries, a very different approach has been used to construct initial data representing neutron stars in quasi-circular orbits and to locate the ISCO. At the core of this approach is the assumption that the space-time admits an approximate Killing vector, $\vec{\ell} \equiv(\partial / \partial t)+\Omega(\partial / \partial \phi)$. This approach was first tested for the case of a single spinning neutron star[12]. Subsequently, variations of this method were used to study neutron-star binaries in quasi-circular orbits 13, 14, 15, 16, 17, 18, 19, 20]. This approach for generating initial data for neutron-star binaries in quasicircular orbits is particularly attractive when compared to the effective potential method used for black-hole binaries. First, if the neutron-star matter is to be in hydrostatic equilibrium, then the 4-velocity of the matter must be proportional to the approximate Killing vector, $\vec{\ell}$. The assumption that $\vec{\ell}$ is a Killing vector ultimately yields a relativistic Bernoulli equation that governs the behavior of the matter and, most importantly, yields a unique value of the angular velocity, $\Omega$, of the binary system in a circular orbit at a given separation. The presence of matter also sets a natural length scale for the the problem and conservation of baryon number makes it straightforward to connect initial-data solutions at different radii to produce meaningful evolutionary sequences.

Recently, Gourgoulhon et al. 21, 22, (hereafter GGB1 and GGB2 respectively) have attempted to adapt the approach used to construct neutron-star binary initial data to the case of black-hole binaries. Their initial results are very encouraging, showing much better agreement with post-Newtonian techniques for locating the ISCO. However, there seem to be problems with their solutions. It seems likely that their initial data represent a much better approximation to astrophysically realistic black-hole binaries in quasi-circular orbit than the data produced using the effective potential technique. However, these new solutions do not represent valid general relativistic initial data because they do not satisfy the constraint equations everywhere. Furthermore, as I will justify later, I do not believe that the particular problem being solved in GGB1,2 admits a solution. Interestingly, this author had attempted to solve virtually the identical problem 23 but was unable to obtain convergence for the coupled system of equations. As the authors point out in GGB2, they must regularize their solution for the shift in order to obtain an extrinsic curvature that does not diverge at the throats of the black holes. It is this regularization technique that prohibits their solutions from satisfying the constraints everywhere. Although I have concerns regarding the validity of the particular solutions found in GGB2, I believe that the authors of GGB1 may have made a significant contribution to the effort to construct astrophysically realistic black-hole binary initial data. This contribution is rooted in their approach for determining the orbital angular velocity, $\Omega$.

The problem with the approach taken in GGB1 is that it is not directly applicable outside the restrictions of conformal flatness and maximal slicing, and it seems that a solution within these limits does not exist. The limitations in the GGB1 method are rooted in the boundary conditions posed on the inner boundaries of their solution domain, that is, the black-hole boundaries. In GGB1 the authors choose to impose a particular isometry condition on the spatial hypersurface. This isometry condition naturally yields boundary conditions on the throats of the black holes, however, this isometry condition and the boundary conditions derived from it have no direct connection to the desired quasi-equilibrium state of the solution. Interestingly, the authors of GGB1 choose to mix the isometry-derived boundary conditions with a Killinghorizon condition that is essentially an equilibrium condition on the black hole horizon. However, this mixed set of boundary conditions is not consistent with the isometry condition unless the artificial regularization of the shift is performed. Again, this regularization prohibits the solutions from satisfying the constraints everywhere.

In this paper, I will develop a formalism for constructing completely general quasi-equilibrium black hole binary initial data. This formalism is also based on a generalization of the approach used for constructing neutronstar binary initial data. The key to this formalism is a set of boundary conditions that are based on the approximation that the black holes are in quasi-equilibrium. Boundary conditions for all of the quantities required to construct quasi-equilibrium data can be found based on the quasi-equilibrium assumption. These boundary conditions are completely general. In particular, they do not depend on the often-used assumptions of conformal flatness and maximal slicing. This formalism is completed by using the approach developed by GGB1 to determine the orbital angular velocity $\Omega$ of the system, an approach that is also rooted in the assumption of quasi-equilibrium.

In the following sections, I will derive and describe this new formalism. Where appropriate, I include comparisons with related methods in order to clarify the advantages of the new formalism. In $\S$ II, I begin with a general discussion of the decomposition of Einstein's equations and how the initial-data equations are posed. In $\S$ III, I discuss two particular decompositions of the constraint equations. In $\S$ IV, I discuss the strengths and weaknesses of the approach used in GGB1,2 and derive the boundary conditions required for the new formalism. In $\S$, I work through the remaining issues involved in constructing quasi-equilibrium initial data. And in $\S \mathrm{VI}$, I conclude with discussion that focuses on the impact of possible choices for the conformal 3-geometry and slicing condition. 


\section{THE INITIAL-DATA EQUATIONS}

In this work, we will use the standard $3+1$ decomposition with the interval written as

$$
\mathrm{d} s^{2}=-\alpha^{2} \mathrm{~d} t^{2}+\gamma_{i j}\left(\mathrm{~d} x^{i}+\beta^{i} \mathrm{~d} t\right)\left(\mathrm{d} x^{j}+\beta^{j} \mathrm{~d} t\right) .
$$

where $\gamma_{i j}$ is the 3 -metric induced on a $t=$ const. spatial hypersurface, $\alpha$ is the lapse function, and $\beta^{i}$ the shift vector. The extrinsic curvature of the spatial slice, $K_{i j}$, is defined by ${ }^{1}$

$$
K_{i j} \equiv-\frac{1}{2} \mathcal{L}_{n} \gamma_{i j}
$$

where $\mathcal{L}_{n}$ denotes the Lie derivative along the unit normal to the spatial slice, $n^{\mu}$. Einstein's equations, $G_{\mu \nu}=8 \pi T_{\mu \nu}$, then reduce to four sets of equations. Two are evolution equations for the spatial metric and extrinsic curvature:

$$
\partial_{t} \gamma_{i j}=-2 \alpha K_{i j}+\bar{\nabla}_{i} \beta_{j}+\bar{\nabla}_{j} \beta_{i}
$$

and

$$
\begin{aligned}
\partial_{t} K_{i j}=\alpha & \alpha \bar{R}_{i j}-2 K_{i \ell} K_{j}^{\ell}+K K_{i j} \\
& \left.-8 \pi S_{i j}+4 \pi \gamma_{i j}(S-\rho)\right] \\
& -\bar{\nabla}_{i} \bar{\nabla}_{j} \alpha+\beta^{\ell} \bar{\nabla}_{\ell} K_{i j}+K_{i \ell} \bar{\nabla}_{j} \beta^{\ell}+K_{j \ell} \bar{\nabla}_{i} \beta^{\ell} .
\end{aligned}
$$

The remaining two are the constraint equations:

$$
\bar{R}+K^{2}-K_{i j} K^{i j}=16 \pi \rho
$$

and

$$
\bar{\nabla}_{j}\left(K^{i j}-\gamma^{i j} K\right)=8 \pi j^{i}
$$

Here, $\bar{\nabla}_{i}, \bar{R}_{i j}$, and $\bar{R}$ are, respectively, the covariant derivative, Ricci tensor, and Ricci scalar associated with the spatial metric $\gamma_{i j} . K \equiv K_{i}^{i}, S_{i j}$ is the matter stress tensor, $S \equiv S_{i}^{i}, \rho$ is the matter energy density, and $j^{i}$ is the matter momentum density. These matter terms are related to the stress-energy tensor via:

$$
\begin{aligned}
S_{\mu \nu} & \equiv \gamma_{\mu}^{\alpha} \gamma_{\nu}^{\beta} T_{\alpha \beta}, \\
j_{\mu} & \equiv-\gamma_{\mu}^{\nu} n^{\alpha} T_{\nu \alpha}, \\
\rho & \equiv n^{\mu} n^{\nu} T_{\mu \nu} \\
T_{\mu \nu} & =S_{\mu \nu}+2 n_{(\mu} j_{\nu)}+n_{\mu} n_{\nu} \rho .
\end{aligned}
$$

Initial data for a Cauchy evolution via Einstein's equations consists of specifying $\gamma_{i j}$ and $K_{i j}$ on an initial hypersurface. The Hamiltonian constraint (5) and the momentum constraints (6) constitute the initial-data equations of general relativity, and the initial data must satisfy these equations. Equations (5) and (6) constrain four

\footnotetext{
1 In this paper, latin indices denote 3-dimensional spatial indices. Greek indices are 4-dimensional. In $\S$ IV C, latin indices can be replaced with indices varying over the two dimensions of the surface whenever only 2-dimensional tensors are involved in the expression.
}

of the 12 degrees of freedom found in the initial data, $\gamma_{i j}$ and $K_{i j}$. The covariant nature of Einstein's equations allows us four gauge degrees of freedom. The remaining four degrees of freedom are the dynamical degrees of freedom and are split two apiece between the metric and extrinsic curvature.

The task of constructing initial data for a Cauchy evolution via Einstein's equations can be broken down into a few fundamental task shared by any approach.

1. A decomposition of the constraints must be chosen that specifies how the constrained, gauge, and dynamical degrees of freedom are associated with the initial data.

2. Boundary conditions must be chosen for the constrained degrees of freedom such that a) the constraint equations reduce to a well-posed set of elliptic PDEs and b) they are compatible with the desired physical content of the initial data.

3. A choice for the spatial and temporal gauge must be made that is compatible with the desired physical content of the initial data.

4. The dynamical degrees of freedom must be specified so as to yield the desired physical content in the initial data.

Once these steps have been completed, all that remains are the technical aspects of solving the coupled set of elliptic PDEs. The physics lies entirely in the four enumerated steps given above.

\section{DECOMPOSITIONS OF THE CONSTRAINTS}

In the weak field limit where Einstein's equations can be linearized, there are clear ways to determine which components of the initial data are dynamic, which are constrained, and which are gauge. However, in the full nonlinear theory, there is no unique way to perform the decomposition. Perhaps the most widely used class of constraint decompositions are the York-Lichnerowicz conformal decompositions which are based on a conformal decomposition of the metric and various other quantities 24, 25, 26.

The metric is decomposed into a conformal factor, $\psi$, multiplying a conformal metric:

$$
\gamma_{i j} \equiv \psi^{4} \tilde{\gamma}_{i j}
$$

Using (11), the Hamiltonian constraint (5) can be written as

$$
\tilde{\nabla}^{2} \psi-\frac{1}{8} \psi \tilde{R}-\frac{1}{8} \psi^{5} K^{2}+\frac{1}{8} \psi^{5} K_{i j} K^{i j}=-2 \pi \psi^{5} \rho,
$$

where $\tilde{\nabla}^{2} \equiv \tilde{\nabla}^{i} \tilde{\nabla}_{i}$, and $\tilde{\nabla}_{i}$ and $\tilde{R}$ are the covariant derivative and Ricci scalar associated with $\tilde{\gamma}_{i j}$. With 
this conformal decomposition, the conformal metric, $\tilde{\gamma}_{i j}$ is considered to encode the dynamical degrees of freedom of the metric and the initial gauge choices for the three spatial coordinates. The conformal factor, $\psi$, is the constrained portion of the metric.

Most decompositions of the extrinsic curvature begin by splitting it into its trace, $K$ and tracefree parts, $A_{i j}$,

$$
K_{i j} \equiv A_{i j}+\frac{1}{3} \gamma_{i j} K
$$

The decomposition proceeds by using the fact that we can covariantly split any symmetric tracefree tensor, $\mathcal{S}^{i j}$, as follows [27]:

$$
\mathcal{S}^{i j} \equiv(\mathbb{L} X)^{i j}+\mathcal{T}^{i j}
$$

Here, $\mathcal{T}^{i j}$ is a symmetric, transverse-traceless tensor (i.e., $\nabla_{j} \mathcal{T}^{i j}=0$ and $\mathcal{T}_{i}^{i}=0$ ) and, in three dimensions,

$$
(\mathbb{L} X)^{i j} \equiv \nabla^{i} X^{j}+\nabla^{j} X^{i}-\frac{2}{3} \gamma^{i j} \nabla_{\ell} X^{\ell} .
$$

At this point, the decomposition can proceed in several different directions, and we will consider two of them below. However, in all cases, $K$ is considered to encode the initial temporal gauge choice, $\mathcal{T}^{i j}$ encodes the two dynamical degrees of freedom, and $X^{i}$ are the three constrained degrees of freedom.

\section{A. Conformal transverse-traceless decomposition}

Historically, the most widely used decomposition expresses the extrinsic curvature as

$$
K^{i j} \equiv \psi^{-10}\left[(\tilde{\mathbb{L}} X)^{i j}+\tilde{Q}^{i j}\right]+\frac{1}{3} \gamma_{i j} K
$$

In this case, the transverse-traceless decomposition takes place with respect to the conformal metric. It will also be convenient to define a conformal trace-free extrinsic curvature, $\tilde{A}^{i j}$, by

$$
\tilde{A}^{i j} \equiv \psi^{10} A^{i j}=(\tilde{\mathbb{L}} X)^{i j}+\tilde{Q}^{i j} .
$$

Using (16) in the momentum constraint (6) yields

$$
\tilde{\Delta}_{\mathbb{L}} X^{i}=\frac{2}{3} \psi^{6} \tilde{\nabla}^{i} K+8 \pi \psi^{10} j^{i}
$$

where

$$
\begin{aligned}
\tilde{\Delta}_{\mathbb{L}} X^{i} & \equiv \tilde{\nabla}_{j}(\tilde{\mathbb{L}} X)^{i j} \\
& =\tilde{\nabla}^{2} X^{i}+\frac{1}{3} \tilde{\nabla}^{i}\left(\tilde{\nabla}_{j} X^{j}\right)+\tilde{R}_{j}^{i} X^{j},
\end{aligned}
$$

and $\tilde{R}_{j}^{i}$ is the Ricci tensor associated with $\tilde{\gamma}_{i j}$. In terms of (17), the Hamiltonian constrain (12) becomes

$$
\begin{aligned}
& \tilde{\nabla}^{2} \psi-\frac{1}{8} \psi \tilde{R}-\frac{1}{12} \psi^{5} K^{2} \\
&+\frac{1}{8} \psi^{-7} \tilde{A}_{i j} \tilde{A}^{i j}=-2 \pi \psi^{5} \rho
\end{aligned}
$$

Together, Eqns. (11) and (16)-(20) constitute the full conformal transverse-traceless decomposition of the constraints. To solve this set of equations, one must specify $\tilde{\gamma}_{i j}, \tilde{Q}^{i j}$, and $K$ in addition to the matter terms $\rho$ and $j^{i}$.

The reason that this decomposition has been so widely used stems from the simplifications that occur when the freely specifiable data are chosen so that the initial data hypersurface is maximal, $K=0$, conformally flat so that $\tilde{\gamma}_{i j}$ is a flat metric, and $\tilde{Q}^{i j}$ is chosen to vanish. Then, for vacuum spacetimes, the constraints reduce to

$$
\tilde{\nabla}^{2} \psi+\frac{1}{8} \psi^{-7} \tilde{A}_{i j} \tilde{A}^{i j}=0,
$$

and

$$
\tilde{\nabla}^{2} X^{i}+\frac{1}{3} \tilde{\nabla}^{i}\left(\tilde{\nabla}_{j} X^{j}\right)=0
$$

and $\tilde{\nabla}_{i}$ is, in this case, the usual flat space covariant derivative operator. What is remarkable about this simplified system of equations is that Eq. (22) decouples completely from (21) and has analytic solutions that represent a single black hole with any desired value of linear and angular momentum 8,28 . Furthermore, since (22) is linear, any number of these solutions may be superposed to represent multiple black holes. All that is required to construct complete initial data is to solve the single quasi-linear elliptic PDE in Eq. 21).

All of the work to date that has used the effective potential method to locate quasi-circular orbits in blackhole binary systems 3, 4, 5] has used initial data computed by this system. The only difference between the data used in Refs. [3, 5], and in Ref. [4] is in the topology of the initial data hypersurfaces.

\section{B. Conformal thin-sandwich decomposition}

An alternative decomposition recently proposed by York 29 is a generalization of an approach used first by Wilson 30] and later by others 12, 13, 31 for the study of neutron-star binaries. Furthermore, aside from some trivial differences in conformal scalings, it also subsumes the approach outlined in GGB1. This decomposition differs from the conformal transverse-traceless decomposition significantly in philosophy, but is very similar in form. The difference is found in the decomposition of the extrinsic curvature. In the conformal thin-sandwich decomposition, $K_{i j}$ is decomposed as

$$
K^{i j}=\frac{\psi^{-10}}{2 \tilde{\alpha}}\left((\tilde{\mathbb{L}} \beta)^{i j}-\tilde{u}^{i j}\right)+\frac{1}{3} \gamma_{i j} K,
$$

or

$$
\tilde{A}^{i j}=\frac{1}{2 \tilde{\alpha}}\left((\tilde{\mathbb{L}} \beta)^{i j}-\tilde{u}^{i j}\right)
$$

Here, $\tilde{u}^{i j}$ takes the place of $\tilde{Q}^{i j}$ in representing the freely specifiable dynamical portion of the extrinsic curvature. However, owing to the nature of the thin sandwich decomposition, it also has a more physical meaning as we 
shall see in a moment. Notice that in Eq. (24), it is not the conformal trace-free extrinsic curvature that is decomposed, but rather $2 \tilde{\alpha} \tilde{A}^{i j}$. The new scalar, $\tilde{\alpha}$, is the conformal lapse defined by

$$
\alpha \equiv \psi^{6} \tilde{\alpha}
$$

Also notice that, in the transverse-traceless decomposition, the vector $X^{i}$ has been replaced by the shift vector, $\beta^{i}$.

Using the thin-sandwich decomposition (23) in the momentum constraint (6) yields

$$
\begin{aligned}
\tilde{\Delta}_{\mathbb{L}} \beta^{i}-(\tilde{\mathbb{L}} \beta)^{i j} \tilde{\nabla}_{j} \ln \tilde{\alpha}= & \frac{4}{3} \tilde{\alpha} \psi^{6} \tilde{\nabla}^{i} K \\
& +\tilde{\alpha} \tilde{\nabla}_{j}\left(\frac{1}{\tilde{\alpha}} \tilde{u}^{i j}\right)+16 \pi \tilde{\alpha} \psi^{10} j^{i} .
\end{aligned}
$$

Taken together, Eqns. (11), (23), (24), (26), (19), and (20) constitute the full thin-sandwich decomposition of the constraints. To solve this set of equations, one must specify $\tilde{\gamma}_{i j}, \tilde{u}^{i j}, K$, and $\tilde{\alpha}$ in addition to the matter terms $\rho$ and $j^{i}$.

The thin-sandwich decomposition differs from most initial-data decompositions in that its derivation uses a second spatial hypersurface that is infinitesimally separated from the primary spatial hypersurface. The time and spatial coordinates in these two hypersurfaces are connected by the kinematical variables, $\alpha$ and $\beta^{i}$, and this connection allows us to obtain a physical interpretation for $\tilde{u}^{i j}$, the freely specifiable dynamical portion of the extrinsic curvature. The result is that $\tilde{u}^{i j}$ is a symmetric, tracefree tensor with the physical interpretation that

$$
\tilde{u}_{i j} \equiv \partial_{t} \tilde{\gamma}_{i j},
$$

and $t$ is the time coordinate associated with the time-like vector $t^{\mu}$ that connects points in the two adjacent hypersurfaces that carry the same spatial coordinate labels, i.e.

$$
t^{\mu} \equiv \alpha n^{\mu}+\beta^{\mu}
$$

and $n^{\mu}$ is the timelike unit normal to the primary spatial hypersurface.

This decomposition has proven useful for constructing quasi-equilibrium initial data sets because, in the frame where the configuration is assumed to be timeindependent (the corotating frame), it is a natural approximation to set $\tilde{u}_{i j}=0$. To my knowledge, all of the work so far (including Refs. 112, 13, 16, 30, 31], and GGB1,2) use the same set of assumptions to fix the freely specifiable data in the thin-sandwich decomposition. In particular, they all choose the initial data hypersurface to be maximal, $K=0$, conformally flat so that $\gamma_{i j}$ is a flat metric, $\tilde{u}_{i j}$ is chosen to be zero, and they all fix the lapse by demanding that $\partial_{t} K=0$. The final assumption is a reasonable one since a quasi-equilibrium solution was the goal of each of these works and the time vector (28) is the one corresponding to the corotating frame.
This conformal thin-sandwich decomposition will form the foundation of the initial-data formalism being developed. However, unlike previous works, we will only make the restriction that $\tilde{u}_{i j}=0$. In particular, we will not restrict the formalism to conformally flat maximal slices of the full spacetime, and we will require that all boundary conditions and any further restrictions apply (at least in principle) to general slicings and conformal 3-geometries.

\section{BOUNDARY CONDITIONS FOR THE CONSTRAINT EQUATIONS}

\section{A. The GGB approach}

We now return to the specific problem of using the thin-sandwich decomposition to construct initial data for a pair of black holes in quasi-circular orbits. In addition to the standard assumptions outlined at the end of $\S$ IIIB, GGB1 also makes the assumption that the initial-data hypersurface is inversion symmetric. That is, the initial data hypersurface is taken to consist of two asymptotically flat hypersurfaces that are joined together at the throats of each black hole, and are isometric to each other with each throat being a fixed point set of the isometry condition. The isometry condition then imposes a set of boundary conditions at the throats on all quantities on the manifold. There are actually two choices for the boundary conditions, and GGB1 make the choice that requires the lapse to vanish on the throats. For the shift vector, the isometry imposes the following boundary conditions

$$
\begin{aligned}
\left.\beta^{r}\right|_{\mathcal{S}} & =0, \\
\left.\frac{\partial \beta^{\theta}}{\partial_{r}}\right|_{\mathcal{S}} & =0, \\
\left.\frac{\partial \beta^{\phi}}{\partial_{r}}\right|_{\mathcal{S}} & =0,
\end{aligned}
$$

where we are assuming the throat is a coordinate sphere and $\left.\right|_{\mathcal{S}}$ denotes evaluation on the throat.

Since this version of inversion symmetry requires that $\left.\alpha\right|_{\mathcal{S}}=0$, regularity of the extrinsic curvature at the throats requires

$$
\left.(\tilde{\mathbb{L}} \beta)^{i j}\right|_{\mathcal{S}}=0 .
$$

As GGB1 point out, this condition is potentially problematical.

Given the choice for the isometry condition used in GGB1, it follows 32 that the throats are apparent horizons. GGB1 make the interesting choice of using this fact to change boundary conditions (30) and (31) on the shift. They obtain new boundary conditions on the shift by demanding that $\partial / \partial_{t}$ be null on the throats so that the throats are Killing horizons. For the case that the lapse vanishes on the throats, this condition yields

$$
\left.\beta^{i}\right|_{\mathcal{S}}=0 \text {. }
$$


Altering the boundary conditions on the shift in this way poses a problem. Using Eq. (33) to replace conditions (30) and (31) means that the solution is not guaranteed to be inversion-symmetric. This, in turn, means that the inner boundaries are not guaranteed to be apparent horizons, from which it follows the Killing horizon boundary conditions may not be appropriate for the inner boundaries.

In GGB1, the authors go to great length to show that inversion-symmetry, their Killing-horizon boundary conditions on the shift, and the required regularity condition (32) can be compatible. However, satisfying the Killinghorizon boundary conditions together with some of the inversion-symmetry boundary conditions does not guarantee that the regularity condition (32) is satisfied, or that the resulting solution is inversion symmetric. If fact, as pointed out in GGB2, it seems that their solutions are not compatible with this condition without some kind of artificial regularization of their solution for the shift. This is not surprising. We can rewrite the evolution equation for the metric as

$$
\begin{aligned}
\partial_{t} \gamma_{i j} & =u_{i j}+\frac{2}{3} \gamma_{i j}\left(\bar{\nabla}_{k} \beta^{k}-\alpha K\right) \\
& =\psi^{4}\left[\tilde{u}_{i j}+\frac{2}{3} \tilde{\gamma}_{i j}\left(\tilde{\nabla}_{k} \beta^{k}+6 \beta^{k} \tilde{\nabla}_{k} \ln \psi-\psi^{6} \tilde{\alpha} K\right)\right],
\end{aligned}
$$

with $u_{i j} \equiv \psi^{4} \tilde{u}_{i j}$. Given the the Killing-horizon boundary conditions and the choices of maximal slicing and $\tilde{u}_{i j}=0$, Eq. (34) reduces to

$$
\left.\partial_{t} \gamma_{i j}\right|_{\mathcal{S}}=\left.\frac{2}{3} \psi^{4} \tilde{\gamma}_{i j} \frac{\partial \beta^{r}}{\partial_{r}}\right|_{\mathcal{S}}
$$

It is demonstrated in GGB1 that regularity requires $\partial \beta^{r} /\left.\partial_{r}\right|_{\mathcal{S}}=0$ and the authors take Eq. (35) as establishing regularity. However, the choice $\tilde{u}_{i j}=0$ is not equivalent to $\partial_{t} \gamma_{i j}=0$, but only fixes the time rate of change of the conformal metric. The time rate of change of the conformal factor is not fixed by this condition and is only guaranteed to vanish if $\vec{t}$ is a true Killing vector. Thus, for a quasi-equilibrium solution of the constraints, it seems reasonable that $\partial \beta^{r} /\left.\partial_{r}\right|_{\mathcal{S}}$ is small in some appropriate norm, but it is unlikely to vanish.

So, the approach outlined in GGB1 has two technical problems: the boundary conditions being used do not guarantee that the solution is inversion symmetric or that the solution will be regular at the inner boundary. Both of these problems are fixed in GGB2 by an artificial regularization of the shift solution. The unfortunate side effect of this regularization procedure is that the solutions no longer satisfy the constraint equations and, thus, cannot represent valid initial data. In fact, it seems that a regular, inversion-symmetric solution for the extrinsic curvature is not possible (or is at least extremely unlikely), given the assumptions of GGB1.

\section{B. Killing-horizon boundary conditions}

Although the particular set of boundary conditions proposed in GGB1 does not seem to yield a well-posed system of elliptic equations, the idea of using some kind of boundary conditions on the apparent horizons of the black holes is promising. An alternate approach for obtaining boundary conditions on the shift is found by a slight generalization of the the Killing-horizon conditions proposed in GGB1. As we will see, this change in perspective will allow us to derive boundary conditions on the shift that will yield corotating black holes, irrotational black holes, or black holes with some arbitrary angular velocity. We begin by noting that the outward pointing null vector whose vanishing expansion defines the apparent horizon can be written as

$$
k^{\mu} \propto\left(n^{\mu}+s^{\mu}\right) .
$$

Here, $s^{\mu}$ is the outward pointing unit vector normal to the apparent horizon (see $\S \mathbb{I V G}$ for further details). In terms of components, this can be written as

$$
k^{\mu}=\left[1, \alpha s^{i}-\beta^{i}\right],
$$

where I have chosen a normalization so that $k^{t} \equiv 1$. Note that $k^{\mu}$ is null by construction and $k^{\mu} k_{\mu}=0$ for any choice of the shift vector.

If we assume rotation in the $\phi$ direction, then the approximate killing vector for a quasi-equilibrium configuration is given by

$$
\vec{\ell} \equiv \frac{\partial}{\partial t}+\Omega \frac{\partial}{\partial \phi}
$$

where $\Omega$ is the orbital angular velocity of the binary, and $\partial / \partial \phi$ represent the asymptotic Killing vector for rotation in the $\phi$ direction. In the corotating coordinate system, $\vec{\ell}$ is the time vector and it has components $\ell^{\mu}=[1,0,0,0]$. If the individual black holes are corotating with the system, then they do not appear to be rotating in that coordinate system. In this case, the Killing-horizon condition implies that $\ell^{\mu}$ should be parallel to $k^{\mu}$ which immediately yields

$$
\left.\beta^{i}\right|_{\mathcal{S}}=\left.\alpha s^{i}\right|_{\mathcal{S}}
$$

Notice that this condition reduces directly to Eq. (33) when $\left.\alpha\right|_{\mathcal{S}}=0$.

We can also derive a boundary condition for black holes that are not rotating with respect to observers at rest at infinity, black holes that we might loosely refer to as being irrotational. In this case, the outward pointing null vectors at the apparent horizon should be parallel to $\vec{t}$, not to $\vec{\ell}$. In the corotating coordinate system, the components of $\vec{t}$ can be written symbolically as

$$
t^{\mu}=\left[1,-\Omega(\partial / \partial \phi)^{i}\right]
$$


It follows immediately that the appropriate boundary conditions for irrotational black holes are

$$
\left.\beta^{i}\right|_{\mathcal{S}}=\left.\alpha s^{i}\right|_{\mathcal{S}}+\left.\Omega\left(\frac{\partial}{\partial \phi}\right)^{i}\right|_{\mathcal{S}} .
$$

For both boundary conditions, corotation given by (39), and irrotational given by (41), the conditions are given in terms of the corotating coordinate system. This means that the appropriate asymptotic boundary conditions for the shift are that

$$
\left.\beta^{i}\right|_{\mathrm{r} \rightarrow \infty} \sim \Omega\left(\frac{\partial}{\partial \phi}\right)^{i} .
$$

The corotation and irrotational conditions can be generalized to yield a boundary condition for an equilibrium black holes with arbitrary spin. Let $\chi^{i}$ denote a flat-space Killing vector for rotation in an arbitrary direction, just as $(\partial / \partial \phi)^{i}$ represents rotation about in the $\phi$ direction. With $\Omega_{\chi}$ representing the angular velocity of rotation of the black holes as measured by observers at rest at infinity, we find that the components of the null vectors generating the horizon are

$$
\left[1, \Omega_{\chi} \chi^{i}-\Omega(\partial / \partial \phi)^{i}\right]
$$

when written in terms of the corotating coordinate system. This immediately yields the boundary conditions for a quasi-equilibrium black hole with arbitrary spin:

$$
\left.\beta^{i}\right|_{\mathcal{S}}=\left.\alpha s^{i}\right|_{\mathcal{S}}-\left.\Omega_{\chi} \chi^{i}\right|_{\mathcal{S}}+\left.\Omega\left(\frac{\partial}{\partial \phi}\right)^{i}\right|_{\mathcal{S}} .
$$

As we will see below, we will need to be a bit more careful in defining the shift boundary condition for the irrotational and general spin cases, but (41) and (44) give us a good starting point for these conditions.

\section{Quasi-equilibrium boundary conditions}

The choice of the particular inversion-symmetric class of black-hole initial data used in GGB1 was made because it guarantees that the inner boundary surfaces will be apparent horizons. If we are to give up inversion-symmetry, we must find some other way of fixing boundary conditions at the inner-boundary surfaces for all of the quantities needed to construct initial data. These boundary conditions can be found by forcing the inner boundary to be an apparent horizon and by assuming that the associated black hole is in quasi-equilibrium. Pieces of the resulting approach have been worked out by Thornburg [33] and by Eardley 34], but to my knowledge have not been adapted for constructing quasi-equilibrium initial data. Because there are several errors in the derivations and results in Ref. [34, and to provide a uniform notation, I will rederive all of the equations here.
Our inner boundary surface, $\mathcal{S}$, is assumed to be a spacelike 2 -surface with topology $S^{2}$. Because $\mathcal{S}$ is closed, we can define its unit normal via

$$
s_{i} \equiv \lambda \bar{\nabla}_{i} \tau
$$

where $\lambda$ is a normalization constant fixed by $s^{i} s_{i} \equiv 1$ and $\tau$ is a scalar function which has a level surface $\tau=0$ that defines the surface $\mathcal{S}$. The 4-dimensional generalization of $s^{i}$ has components $s^{\mu}=\left[0, s^{i}\right]$ obtained from the condition that $s^{\mu} n_{\mu}=0$.

The metric, $h_{i j}$, induced on $\mathcal{S}$ by $\gamma_{i j}$ is given by

$$
h_{i j} \equiv \gamma_{i j}-s_{i} s_{j}
$$

We also define the extrinsic curvature, $H_{i j}$, of $\mathcal{S}$ embedded in the 3-dimensional spatial hypersurface as

$$
H_{i j} \equiv-h_{i}^{k} h_{j}^{\ell} \bar{\nabla}_{(k} s_{\ell)}=-\frac{1}{2} \mathcal{L}_{s} h_{i j} .
$$

Naturally associated with $\mathcal{S}$ are two sets of null vectors: a set of outgoing null rays, $k^{\mu}$, and a set if ingoing null rays, $\dot{k}^{\mu}$, defined by

$$
k^{\mu}=\frac{1}{\sqrt{2}}\left(n^{\mu}+s^{\mu}\right) \quad \text { and } \quad \dot{k}^{\mu}=\frac{1}{\sqrt{2}}\left(n^{\mu}-s^{\mu}\right) .
$$

Associated with each set of null rays is an extrinsic curvature of $\mathcal{S}$ as embedded in the full 4-dimensional manifold. These are defined as

$$
\begin{aligned}
\Sigma_{\mu \nu} & \equiv-\frac{1}{2} h_{\mu}^{\alpha} h_{\nu}^{\beta} \mathcal{L}_{k} g_{\alpha \beta}, \\
\dot{\Sigma}_{\mu \nu} & \equiv-\frac{1}{2} h_{\mu}^{\alpha} h_{\nu}^{\beta} \mathcal{L}_{k} g_{\alpha \beta} .
\end{aligned}
$$

To simplify the following, we will introduce various projections of $K_{i j}$ along and normal to $\mathcal{S}$ :

$$
\begin{aligned}
J_{i j} & \equiv h_{i}^{k} h_{j}^{\ell} K_{k \ell}, \\
J_{i} & \equiv h_{i}^{k} s^{\ell} K_{k \ell}, \\
J & \equiv h^{i j} J_{i j}=h^{i j} K_{i j}, \\
K_{i j} & =J_{i j}+2 s_{(i} J_{j)}+s_{i} s_{j}(K-J) .
\end{aligned}
$$

We can then simplify Eqns. (49) and (50) to

$$
\Sigma_{i j}=\frac{1}{\sqrt{2}}\left(J_{i j}+H_{i j}\right) \quad \text { and } \quad \dot{\Sigma}_{i j}=\frac{1}{\sqrt{2}}\left(J_{i j}-H_{i j}\right)
$$

Now, we define the expansion of outgoing null rays, $\sigma$, and ingoing null rays, $\sigma$, via

$$
\begin{aligned}
\sigma & \equiv h^{i j} \Sigma_{i j}=\frac{1}{\sqrt{2}}(J+H), \\
\dot{\sigma} & \equiv h^{i j} \dot{\Sigma}_{i j}=\frac{1}{\sqrt{2}}(J-H) .
\end{aligned}
$$

Finally, we will define the shear of the outgoing null rays, $\sigma_{i j}$, and ingoing null rays, $\hat{\sigma}_{i j}$, via

$$
\sigma_{i j} \equiv \Sigma_{i j}-\frac{1}{2} h_{i j} \sigma \quad \text { and } \quad \dot{\sigma}_{i j} \equiv \dot{\Sigma}_{i j}-\frac{1}{2} h_{i j} \dot{\sigma} .
$$

In order to generate boundary conditions, we need to make some assumptions. First, we will demand that our 
inner boundary, $\mathcal{S}$, is an apparent horizon. This is equivalent to demanding that the expansion of the outgoing null rays vanishes on $\mathcal{S}$. Thus our first condition is that

$$
\sigma=0
$$

If we are looking for quasi-equilibrium configurations, then we want $\mathcal{S}$ to remain at the same coordinate location in the 3-dimensional hypersurface as time passes. To enforce this condition, it is necessary that $\mathcal{L}_{t} \tau=\partial_{t} \tau=0$, where $\vec{t}$ is the approximate Killing vector associated with our demand of quasi-equilibrium. However, there is no reason that coordinates cannot be free to move on $\mathcal{S}$. In fact, this freedom is necessary to allow for rotation of the black hole. This means that, in order to keep $\mathcal{S}$ at the same coordinate location, $\partial_{t} \tau=0$ is too strong a condition. If we define $\vec{\zeta}$ as the part of $\vec{t}$ that is orthogonal to $\mathcal{S}$, then it is necessary and sufficient that

$$
\mathcal{L}_{\zeta} \tau=0 \quad \text { and } \quad h_{i}^{j} \bar{\nabla}_{j} \mathcal{L}_{\zeta} \tau=\hat{\nabla}_{i} \mathcal{L}_{\zeta} \tau=0,
$$

where $\hat{\nabla}_{i}$ is the 2-dimensional covariant derivative compatible with $h_{i j}$. If we define the normal component of the shift as

$$
\beta_{\perp} \equiv \beta^{i} s_{i}
$$

then we can write $\vec{\zeta}$ as

$$
\zeta^{\mu} \equiv \alpha n^{\mu}+\beta_{\perp} s^{\mu}
$$

We now need to consider how the expansions, $\sigma$ and $\hat{\sigma}$, evolve along $\vec{\zeta}$. For this calculation, a few identities are crucial:

$$
\begin{aligned}
h^{i j} \bar{R}_{i j} & =\frac{1}{2}\left(\bar{R}-H^{2}+H_{i j} H^{i j}+\hat{R}\right), \\
s^{i} s^{j} \bar{R}_{i j} & =\frac{1}{2}\left(\bar{R}+H^{2}-H_{i j} H^{i j}-\hat{R}\right), \\
\lambda\left[\mathcal{L}_{\zeta}, \bar{\nabla}_{\mu}\right] \tau & =-n_{\mu}\left[n^{\nu} \nabla_{\nu} \beta_{\perp}+\beta_{\perp}(K-J)\right],
\end{aligned}
$$

where $\hat{R}$ is the Ricci scalar associated with $h_{i j}$ and $\nabla_{\mu}$ is the 4-dimensional covariant derivative. The first two identities are obtained from various combinations and contractions of the Gauss-Codazzi-Ricci equations that govern the embedding of $\mathcal{S}$ in the the 3-dimensional hypersurface. The third identity can be obtained by direct calculation.

As useful intermediate results, we obtain:

$$
\begin{aligned}
\mathcal{L}_{\zeta} J= & {\left[\frac{1}{2}\left(J^{2}-H^{2}\right)+\frac{1}{2}\left(J_{i j} J^{i j}+H_{i j} H^{i j}\right)\right.} \\
& \left.-J_{i} J^{i}+8 \pi S_{i j} s^{i} s^{j}+\frac{1}{2} \hat{R}-\hat{\nabla}^{2}\right] \alpha \\
& +\left[J_{i j} H^{i j}+J H-H K-8 \pi j_{i} s^{i}\right. \\
& \left.\left.+\hat{\nabla}_{i} J^{i}+2 J^{i} \hat{\nabla}_{i}\right] \beta_{\perp}+H s^{i} \bar{\nabla}_{i} \alpha, \quad 66\right) \\
\mathcal{L}_{\zeta} H= & {\left[J_{i j} H^{i j}-8 \pi j_{i} s^{i}-\hat{\nabla}_{i} J^{i}-2 J^{i} \hat{\nabla}_{i}\right] \alpha } \\
& +\left[\frac{1}{2}\left(J^{2}+H^{2}\right)+\frac{1}{2}\left(J_{i j} J^{i j}+H_{i j} H^{i j}\right)\right. \\
& \left.+J_{i} J^{i}-J K+8 \pi \rho-\frac{1}{2} \hat{R}+\hat{\nabla}^{2}\right] \beta_{\perp} \\
& +J s^{i} \bar{\nabla}_{i} \alpha .
\end{aligned}
$$

These equations follow from the identities $(63)-(65)$ and the evolution and constraint equations (3)-(6). Finally, we can simplify these to the desired results:

$$
\begin{aligned}
& \mathcal{L}_{\zeta} \sigma=\frac{1}{\sqrt{2}}\left[\sigma\left(\sigma+\frac{1}{2} \sigma-\frac{1}{\sqrt{2}} K\right)+\mathcal{E}\right]\left(\beta_{\perp}+\alpha\right) \\
& \frac{1}{\sqrt{2}}\left[\sigma\left(\frac{1}{2} \sigma-\frac{1}{2} \sigma-\frac{1}{\sqrt{2}} K\right)+\mathcal{D}\right. \\
& \left.+8 \pi T_{\mu \nu} k^{\mu} \dot{k}^{\nu}\right]\left(\beta_{\perp}-\alpha\right)+\sigma s^{i} \bar{\nabla}_{i} \alpha, \\
& \mathcal{L}_{\zeta} \dot{\sigma}=-\frac{1}{\sqrt{2}}\left[\dot{\sigma}\left(\dot{\sigma}+\frac{1}{2} \sigma-\frac{1}{\sqrt{2}} K\right)+\dot{\mathcal{E}}\right]\left(\beta_{\perp}-\alpha\right) \\
& -\frac{1}{\sqrt{2}}\left[\dot{\sigma}\left(\frac{1}{2} \dot{\sigma}-\frac{1}{2} \sigma-\frac{1}{\sqrt{2}} K\right)+\dot{\mathcal{D}}\right. \\
& \left.+8 \pi T_{\mu \nu} k^{\mu} \dot{k}^{\nu}\right]\left(\beta_{\perp}+\alpha\right)-\dot{\sigma} s^{i} \bar{\nabla}_{i} \alpha
\end{aligned}
$$

where

$$
\begin{aligned}
\mathcal{D} & \equiv h^{i j}\left(\hat{\nabla}_{i}+J_{i}\right)\left(\hat{\nabla}_{j}+J_{j}\right)-\frac{1}{2} \hat{R} \\
\mathcal{D} & \equiv h^{i j}\left(\hat{\nabla}_{i}-J_{i}\right)\left(\hat{\nabla}_{j}-J_{j}\right)-\frac{1}{2} \hat{R}, \\
\mathcal{E} & \equiv \sigma_{i j} \sigma^{i j}+8 \pi T_{\mu \nu} k^{\mu} k^{\nu}, \\
\dot{\mathcal{E}} & \equiv \dot{\sigma}_{i j} \dot{\sigma}^{i j}+8 \pi T_{\mu \nu} \dot{k}^{\mu} \dot{k}^{\nu} .
\end{aligned}
$$

We note that $\mathcal{E}$ and $\mathcal{E}$ are both non-negative so long as the matter satisfies either the weak or the strong energy condition. The two equations for the evolution of the expansions, (68) and (69), are not yet restricted by any assumption of quasi-equilibrium. The only assumption in their derivation is that the surface $\mathcal{S}$ remain at a constant coordinate location which is embodied in (60).

If we now restrict Eqns. (68) and (69) to apply to apparent horizons by enforcing condition (59) and also impose quasi-equilibrium on the evolution equations for the expansions,

$$
\mathcal{L}_{\zeta} \sigma=0 \quad \text { and } \quad \mathcal{L}_{\zeta} \sigma=0
$$

then we find

$$
\begin{aligned}
0= & \mathcal{D}\left(\beta_{\perp}-\alpha\right) \\
\dot{\sigma}^{i}{ }^{i} \bar{\nabla}_{i} \alpha= & -\frac{1}{\sqrt{2}}\left[\dot{\sigma}\left(\dot{\sigma}-\frac{1}{\sqrt{2}} K\right)+\dot{\sigma}_{i j} \dot{\sigma}^{i j}\right]\left(\beta_{\perp}-\alpha\right) \\
& -\frac{1}{\sqrt{2}}\left[\dot{\sigma}\left(\frac{1}{2} \dot{\sigma}-\frac{1}{\sqrt{2}} K\right)+\dot{\mathcal{D}}\right]\left(\beta_{\perp}+\alpha\right) .
\end{aligned}
$$

These equations are based on the approximation that the shear of the outgoing null rays on the apparent horizon vanishes, which is true for the case of a stationary black hole, and on the requirements that $T_{\mu \nu} k^{\mu} k^{\nu}=$ $T_{\mu \nu} \hat{k}^{\mu} \hat{k}^{\nu}=T_{\mu \nu} k^{\mu} \hat{k}^{\nu}=0$ on the apparent horizon if the black hole is in quasi-equilibrium. Finally, I reemphasize that the conditions for quasi-equilibrium on the two expansions is given by Eq. (74), not by $\partial_{t} \sigma=\partial_{t} \sigma=0$, so that the location of the horizon is fixed but the black hole is allowed to rotate in $\mathcal{S}$.

On inspection, Eq. (75) yields the solution

$$
\beta_{\perp}=\alpha
$$


which is compatible with the Killing horizon boundary condition obtained in the case of corotation (39). The Killing horizon boundary conditions for irrotation and general rotation, (41) and (44), are not compatible with (77) unless we restrict the rotational terms to act in the surface $\mathcal{S}$. Thus, the correct boundary condition on the shift for an irrotational black hole should be

$$
\left.\beta^{i}\right|_{\mathcal{S}}=\left.\alpha s^{i}\right|_{\mathcal{S}}+\left.\Omega h_{j}^{i}\left(\frac{\partial}{\partial \phi}\right)^{j}\right|_{\mathcal{S}},
$$

and the condition for general rotation should be

$$
\left.\beta^{i}\right|_{\mathcal{S}}=\left.\alpha s^{i}\right|_{\mathcal{S}}-h_{j}^{i}\left[\Omega_{\chi} \chi^{j}-\Omega\left(\frac{\partial}{\partial \phi}\right)^{j}\right]_{\mathcal{S}} .
$$

Notice that our assumption of quasi-equilibrium has lead to one condition on the shift, and yet Eqns. (39), (78) and (79) specify three conditions on the shift. This is appropriate since we have made specific choices for the rotation of the black hole, namely corotation, irrotation, or some general rotation specified by $\Omega_{\chi}$ and $\chi^{i}$.

The condition that $\mathcal{S}$ be an apparent horizon, (59), also yields a useful boundary condition if we work in the conformal space. The conformal transformation on $\gamma_{i j}$ (11) induces a natural conformal weighting for $h_{i j}$ and for the unit normal to $\mathcal{S}$,

$$
\begin{aligned}
h_{i j} & \equiv \psi^{4} \tilde{h}_{i j}, \\
s^{i} & \equiv \psi^{-2} \tilde{s}^{i} .
\end{aligned}
$$

We find that Eq. (59) reduces to

$$
\left.\tilde{s}^{k} \tilde{\nabla}_{k} \ln \psi\right|_{\mathcal{S}}=-\left.\frac{1}{4}\left(\tilde{h}^{i j} \tilde{\nabla}_{i} \tilde{s}_{j}-\psi^{2} J\right)\right|_{\mathcal{S}},
$$

which is a non-linear boundary condition that incorporates both $\psi$ and its normal derivative. While this boundary conditions looks somewhat complicated, it reduces to the often used minimal surface boundary condition whenever $\left.J\right|_{\mathcal{S}}=0$, and it has previously been used successfully to construction initial-data sets 33 .

We can now further simplify the remaining condition given in Eq. (76). From Eqns. (56), (57), and (59), we obtain

$$
\dot{\sigma}=\sqrt{2} J .
$$

Together with (77), (80), and (81), this reduces $(76)$ to

$$
\left.J \tilde{s}^{i} \tilde{\nabla}_{i} \alpha\right|_{\mathcal{S}}=-\left.\psi^{2}\left(J^{2}-J K+\tilde{\mathcal{D}}\right) \alpha\right|_{\mathcal{S}},
$$

with

$$
\tilde{\mathcal{D}} \equiv \psi^{-4}\left[\tilde{h}^{i j}\left(\breve{\nabla}_{i}-J_{i}\right)\left(\breve{\nabla}_{j}-J_{j}\right)-\frac{1}{2} \breve{R}+2 \breve{\nabla}^{2} \ln \psi\right] .
$$

Here, $\breve{\nabla}_{i}$ and $\breve{R}$ are the covariant derivative and Ricci scalar associated with the conformal metric $\tilde{h}_{i j}$. Equation (34) represents a complicated mixed condition on the lapse that involves an elliptic operator acting over the closed surface $\mathcal{S}$. There is no guarantee that (85) is invertible, however, if it is, then (84) represents a viable boundary condition.

\section{QUASI-CIRCULAR ORBITS FOR BLACK-HOLE BINARIES}

The primary goal of this paper is to fully define a formalism for constructing initial data sets representing astrophysically realistic black-hole binaries in quasi-circular orbits. As was shown in $\S$ IIIB, the thin-sandwich decomposition of the constraint equations appears to be a natural choice to use for this purpose. In particular, the simple choice of $\tilde{u}^{i j}=0$ is required by the assumption of quasi-equilibrium. In $\S$ IV G, boundary conditions compatible with quasi-equilibrium were developed. However, there are still several freely specifiable quantities that we have not considered within the context of quasi-equilibrium. In particular, we must consider how the initial temporal gauge choice evolves off of the initial slice via $\tilde{\alpha}$. We must decide what value to use for the orbital angular velocity, $\Omega$, in setting the outer-boundary conditions (42). And finally, we must choose the conformal 3-geometry, $\tilde{\gamma}_{i j}$, and the initial temporal gauge via $K$.

\section{A. The temporal gauge choice}

In all previous work on quasi-equilibrium data for either neutron stars or black holes, maximal slicing has been used. With this choice, $K=0$, and it is natural to use the "maximal slicing equation" to fix the lapse. The maximal slicing equation has been used extensively in numerical evolutions and is simply a linear second-order elliptic equation for the lapse derived from the evolution equation for $K$ by setting $\partial_{t} K=0$ (cf. Ref. 35]). For a quasi-equilibrium situation, it is natural to use the condition $\partial_{t} K=0$, but to generalize it to any arbitrary value for $K$. In this case, the equation that results,

$$
\begin{array}{r}
\tilde{\nabla}^{2}(\alpha \psi)-\alpha\left[\frac{1}{8} \psi \tilde{R}+\frac{5}{12} \psi^{5} K^{2}+\frac{7}{8} \psi^{-7} \tilde{A}_{i j} \tilde{A}^{i j}\right. \\
\left.+2 \pi \psi^{5} K(\rho+2 S)\right]=\psi^{5} \beta^{i} \tilde{\nabla}_{i} K
\end{array}
$$

is often called the "constant trace- $K$ equation" (constant refers to the fact that $\mathrm{K}$ is constant in time, not constant in space).

For a quasi-equilibrium situation, we would like to have $\partial_{t} \gamma_{i j}=0$ and $\partial_{t} K_{i j}=0$. However, this will only be obtainable in true equilibrium situations where we have exact Killing vectors. We can decompose these conditions to get $\partial_{t} \tilde{\gamma}_{i j}=0, \partial_{t} \psi=0, \partial_{t} \tilde{A}_{i j}=0$ and $\partial_{t} K=0$ as the possible quasi-equilibrium conditions that we might apply. Of these, only $\partial_{t} \tilde{\gamma}_{i j}=\tilde{u}_{i j}$ is part of the freely specifiable data of the thin-sandwich decomposition. Given that we must fix $\tilde{\gamma}_{i j}$ and that we choose $\tilde{u}_{i j}=0$ as a quasi-equilibrium condition, it is not reasonable to assume that we can also find $\partial_{t} \tilde{A}_{i j}=0$ except in true equilibrium conditions. In addition to two dynamical degrees of freedom, $\tilde{\gamma}_{i j}$ also encodes the initial spatial gauge choice. Similarly, $K$ encodes the initial temporal gauge choice and parity with the spatial gauge suggests that we 
should let quasi-equilibrium fix $\partial_{t} K=0$ and that it is not reasonable to expect $\partial_{t} \psi=0$ except in true equilibrium conditions.

We can examine the condition $\partial_{t} \psi=0$ even further. From Eq. (34) we find that this condition is equivalent to

$$
\tilde{\nabla}_{k} \beta^{k}+6 \beta^{k} \tilde{\nabla}_{k} \ln \psi-\psi^{6} \tilde{\alpha} K=0 .
$$

We might consider using (87) to fix $K$ instead of choosing it freely. However, if we use (87) to replace $K$ in either Eq. (26) or (18) we find that the resulting elliptic operator is non-invertible. One might then hope that an iterative scheme could be used to fix $K$ so that $\partial_{t} \psi=0$ is satisfied. However, numerical experiments with this approach have shown it to be unstable 36.

Given that the thin-sandwich decomposition requires that $\tilde{\alpha}$ be fixed, it is fortunate that the condition of quasiequilibrium provides an elliptic equation that fixes this quantity, just as the condition of quasi-equilibrium provides the means of constructing boundary conditions for the constraints. Furthermore, quasi-equilibrium also provides a boundary condition on $\tilde{\alpha}$ for use in (86). This boundary condition is given by Eq. (84) with $\alpha$ replaced by $\tilde{\alpha}$ via (25). At spatial infinity, we have that $\tilde{\alpha}=1$.

\section{B. Obtaining circular orbits}

Consider solving the thin-sandwich equations, (20) and (26), in conjunction with the constant trace- $K$ equation, (86). Assume that we fix a conformal 3-geometry $\tilde{\gamma}_{i j}$ (not necessarily flat), that we fix $K$ (not necessarily a maximal slice), and that we choose $\tilde{u}^{i j}=0$. We will demand that the apparent horizons associated with each hole in our black-hole binary system occur on specified closed 2surfaces in the given 3-geometry. On these inner boundaries, we will use the conditions given in Eqns. (82), 84), and (39) or (78) together with the asymptotic conditions that $\psi=1, \tilde{\alpha}=1$, and (42). Assuming that the system is well-posed, solving it will result in two one-parameter families of solutions, one for the corotation condition (39) and one for the irrotational condition (78). These families of solutions are parameterized by the orbital angular velocity, $\Omega$.

As is pointed out in GGB1, it is unreasonable to assume that the entire family of solutions will satisfy the physical condition of quasi-equilibrium in which we are interested. This situation is similar to that encountered in searching for quasi-circular orbits via the effective potential method 3]. In fact, the effective potential in that approach was chosen on physical ground to pick those solutions which represented approximately circular orbits. However, that effective potential was necessarily somewhat ad-hoc. In my opinion, the most important contribution of GGB1 is their more physically well-founded and covariant method for determining which members of the family of solutions satisfy the condition of quasiequilibrium.
The condition for choosing appropriate values of $\Omega$ given in GGB1,2 is justified largely in terms of its relation to a general relativistic version of the virial theorem 37 . The condition is that $\Omega$ is chosen so that the standard ADM mass, $E_{\mathrm{ADM}}$, and the Komar(or KVM) mass 38 , $M_{\mathrm{K}}$, agree. In my view, the justification for this condition resonates most clearly when one considers that, in general, $E_{\mathrm{ADM}} \neq M_{\mathrm{K}}$. However, as was shown by Beig 39 (see also Ref. 40]), these two mass measures agree when the spacetime is stationary. Since a quasi-equilibrium solution is approximating a stationary spacetime, it is natural to demand that $E_{\mathrm{ADM}}=M_{\mathrm{K}}$, and in this case, we should obtain quasi-circular orbits.

Since, for a general gauge choice, $E_{\mathrm{ADM}}$ is not necessarily given by the monopole piece of the conformal factor, we will use a general definition of the ADM mass 41,

$$
E_{\mathrm{ADM}}=\frac{1}{16 \pi} \oint_{\infty} \gamma^{i j} \bar{\nabla}_{k}\left(\mathcal{G}_{i}^{k}-\delta_{i}^{k} \mathcal{G}\right) \mathrm{d}^{2} S_{j}
$$

Here, $\mathcal{G}_{i j} \equiv \gamma_{i j}-f_{i j}, f_{i j}$ is the flat metric to which $\gamma_{i j}$ asymptotes, and $\mathrm{d}^{2} S_{i}$ is the covariant surface area element. We note that in (88), indices can be raised and lowered with either $\gamma_{i j}$ or $f_{i j}$, the trace, $\mathcal{G}$, can be obtained with either metric, and $\bar{\nabla}_{i}$ can be replaced with the flat covariant derivative. Similarly, a general definition of the Komar mass can be written as 37

$$
M_{\mathrm{K}}=\frac{1}{4 \pi} \oint_{\infty} \gamma^{i j}\left(\bar{\nabla}_{i} \alpha-\beta^{k} K_{i k}\right) \mathrm{d}^{2} S_{j} .
$$

In many cases, $\beta^{k} K_{i k}$ will fall off faster than $O\left(r^{-2}\right)$ and the second term in 89 will not contribute. However, in some gauges this term is important. An example is the Painlevé-Gullstrand coordinate system (cf. Ref. 42 ).

\section{Quasi-equilibrium evolutionary sequences}

It is highly desirable to be able to construct evolutionary sequences of quasi-equilibrium binary configurations. In particular, this facilitates locating the ISCO. When one considers neutron-star configurations, there is a natural way to connect neighboring solutions. Namely, the number of baryons contained in each star should not change as the star secularly evolves to smaller separation. With black holes, there is no such conserved quantity and one must fix some normalizing condition.

In order to construct evolutionary sequences from quasi-circular orbits obtained by the effective-potential method, both the normalizing condition and the orbital angular velocity had to be determined by some means. One condition that should be applicable to evolutionary sequences of quasi-equilibrium configurations $7,43,44$, 4 is

$$
\Omega=\frac{d E_{\mathrm{ADM}}}{d J} .
$$

This relation has been shown to hold well for evolutionary sequences of both corotating 14 and irrotational 20] 
neutron-star binaries. Because the effective-potential method cannot determine the orbital angular velocity of each configuration of holes in circular orbit, Eq. (90) must be used to determine $\Omega$. But, locating each circularorbit configuration, as well as determining $\Omega$ requires a normalizing condition. In work so far with the effectivepotential method, this normalization condition has been chosen to be an ad hoc definition for the mass of an individual black hole in the binary system, with the further assumption that this mass remains constant as the black holes secularly evolve closer together. While this definition had appropriate limiting behavior when the black holes have large separation, this normalization condition seems problematical in the strong-field limit.

However, because quasi-equilibrium data constructed from the combination of the thin-sandwich and constant trace- $K$ equations naturally yields a value for $\Omega$ for each circular-orbit configuration, GGB2 point out that 90 can be used to set the normalization condition for constructing evolutionary sequences. This appears to be a much more well-founded condition than the ad hoc condition proposed in Ref. [3] for the effective-potential method. That ad hoc condition is essentially rooted in the notion that the area of the apparent horizon, an estimate of the true irreducible mass of a black hole, should remain constant during secular evolution. Interestingly, the first results found in GGB2 show agreement with the ad hoc method to the level of numerical error in the solutions. Given the problems with the solutions obtained in GGB2, as outlined in $\S \mathrm{IVA}$, it is too soon to make any conclusions regarding the validity of the ad hoc condition, but this point should be considered further.

\section{DISCUSSION}

So far, we have not considered specific choices for the conformal 3-geometry, $\tilde{\gamma}_{i j}$, and initial slicing as specified by $K$. To date, only flat conformal 3 -geometries and maximal slicings (I will refer to these as the CFMS assumptions) have been explored extensively. It has been pointed out that quasi-equilibrium binary systems in circular orbits will not exhibit a conformally flat 3-geometry at second post-Newtonian order 45. Some consider this approximation to be the major source of error in current numerical work to model quasi-equilibrium binary configurations and in locating the ISCO [7]. Certainly, the assumption of conformal flatness introduces some error. But, it is not clear that this is the most significant source of error in these calculations. As stated earlier, I suspect that the ad hoc normalization condition used to determine $\Omega$ and construct evolutionary sequences is the dominant source of error. It will be important to explore the sensitivity of solutions to the choices for $\tilde{\gamma}_{i j}$ and $K$ in order to understand this issue better.

Of course, it is possible that no regular quasiequilibrium solutions exist when the CFMS assumptions are made. As discussed in $\S$ IVA, a regular solution for the binary problem does not seem possible when inversion-symmetric boundary conditions are chosen becasue $\left.\alpha\right|_{\mathcal{S}}=0$. If we consider the case of a single black hole, we can easily find a CFMS solution of Eqns. (20), (26), and (86) that satisfies the inner boundary conditions given by Eqns. (77), (82), and (84). This solution is simply the Schwarzschild solution in isotropic coordinates. In this case, although $\alpha=0$ on the apparent horizon, the solution is regular. This is possible because the spacetime admits a true Killing vector. The direct generalization of this solution to a binary system yields the problem attempted in GGB1,2 that does not yield a regular solution. However, this does not mean that a quasi-equilibrium solution is impossible given the CFMS assumptions. The nonlinearity of the system of equations and boundary conditions allow for at least the possibility of such solutions, even if it seems unlikely.

The apparent problems with constructing a quasiequilibrium, binary, CFMS solution should not be taken to suggest that conformally flat quasi-equilibrium binary solutions are unlikely. The problem with the CFMS assumptions is with maximal slicing, not conformal flatness. Again, for the case of a single black hole, we can easily find solutions of the quasi-equilibrium equations and boundary conditions that are conformally flat. One example is obtained from the Schwarzschild solution in Kerr-Schild coordinates, also referred to as ingoing Eddington-Finkelstein coordinates (cf. Ref. 42]). With the radial coordinate transformation

$$
r=\frac{\tilde{r}}{4}\left(1+\sqrt{1+\frac{2 M}{\tilde{r}}}\right)^{2} e^{2\left(1-\sqrt{1+\frac{2 M}{\tilde{r}}}\right)},
$$

where $\tilde{r}$ is the usual areal radial coordinate in Kerr-Schild coordinates, the spatial metric on the Kerr-Schild slicing is seen to be conformally flat. Equations (20), (26), and (86) are obviously satisfied, and it is easy to verify that the quasi-equilibrium boundary conditions given by Eqns. (77), (82), and (84) are also satisfied on the horizon at $\tilde{r}=2 M$. Of course, the slicing in this solution is not maximal, or even asymptotically maximal. A feature of this and other similar non-maximal single hole solutions is that $\alpha \neq 0$ on the horizon. It seems likely that this feature will also hold for similar binary configurations, removing the most obvious obstacle confronting the construction of regular quasi-equilibrium solutions.

It is clear that we will want to explore configurations that are not constructed on a maximal slice and we will want to consider conformal 3-geometries that are not flat. This idea has been explored in similar contexts [10, 11, but little has been done other than to demonstrate that solutions can be found. The major limitation of these works is that the boundary conditions used were not well motivated. However, some of the work done in constructing appropriate values for $\tilde{\gamma}_{i j}$ and $K$ based on the Schwarzschild and Kerr geometries should prove useful. In the context of quasi-equilibrium solutions, one should construct conformal 3-geometries and associated values 
of $K$ that approximate black hole binaries that are at rest in the corotating coordinate system. By superposing two Schwarzschild holes, one would approximate the 3-geometry of a pair of corotating black holes. A superposition of two Kerr holes could approximate the case of irrotational black holes, assuming the spins are chosen correctly. Again, it will be interesting to asses the sensitivity of corotating and irrotational solutions to the choice of the conformal 3-geometry.

An improvement in the choice for $\tilde{\gamma}_{i j}$ and $K$ would come from taking these data from post-Newtonian solutions for binaries in circular-orbits. An appropriate metric could be constructed by omitting radiation damping terms. One possibility would be to revert the effective one-body metric obtained in Ref. 46] to an appropriate two body coordinate system, assuming this can be done. A metric accurate to $2.5 \mathrm{PN}$ order, including spin effects, has been given explicitly in Ref. 47]. Another possibility is found in Ref. 48] where a post-Newtonian metric is matched to two perturbed Schwarzschild metircs. However, it is unclear to me whether or not any of these solutions are immediately applicable. A usable metric must be written in terms of a gauge where the lapse and 3-metric smoothly cross the individual blackhole horizons. In particular, the lapse should not vanish at these horizons.

Finally, we might consider the use of a slicing and 3metric obtained from a post-Newtonian solution where radiation reaction is included. In this case, we could also obtain a non-zero solution for $\tilde{u}^{i j}$. However, we should be cautious in exploring solutions with $\tilde{u}^{i j} \neq 0$ since this violates the principle of quasi-equilibrium. In particular, the condition used to determine the orbital angular velocity, $\Omega$, will most likely not be applicable in this case.

\section{Acknowledgments}

I would like to thank Mark Scheel and Harald Pfeiffer for helpful discussions. This work was supported in part by NSF grant PHY-9988581.
[1] G. B. Cook, M. W. Choptuik, M. R. Dubal, S. Klasky, R. A. Matzner, and S. R. Oliveira, Phys. Rev. D 47, 1471 (1993).

[2] S. Brandt and B. Brügmann, Phys. Rev. Lett. 78, 3606 (1997).

[3] G. B. Cook, Phys. Rev. D 50, 5025 (1994).

[4] T. W. Baumgarte, Phys. Rev. D 62, 024018 (2000).

[5] H. P. Pfeiffer, S. A. Teukolsky, and G. B. Cook, Phys. Rev. D 62, 104018 (2000).

[6] L. E. Kidder, C. M. Will, and A. G. Wiseman, Class. Quantum Gravit. 9, L125 (1992).

[7] T. Damour, P. Jaranowski, and G. Schafer, Phys. Rev. D 62, 084011 (2000).

[8] J. M. Bowen and J. W. York, Jr., Phys. Rev. D 21, 2047 (1980).

[9] A. D. Kulkarni, L. C. Shepley, and J. W. York, Jr., Phys. Lett. 96A, 228 (1983).

[10] R. A. Matzner, M. F. Huq, and D. Shoemaker, Phys. Rev. D 59, 024015 (1999).

[11] P. Marronetti and R. A. Matzner, Phys. Rev. Lett. 85, 5500 (2000).

[12] G. B. Cook, S. L. Shapiro, and S. A. Teukolsky, Phys. Rev. D 53, 5533 (1996).

[13] T. W. Baumgarte, G. B. Cook, M. A. Scheel, S. L. Shapiro, and S. A. Teukolsky, Phys. Rev. Lett. 79, 1182 (1997).

[14] T. W. Baumgarte, G. B. Cook, M. A. Scheel, S. L. Shapiro, and S. A. Teukolsky, Phys. Rev. D 57, 7299 (1998).

[15] T. W. Baumgarte, G. B. Cook, M. A. Scheel, S. L. Shapiro, and S. A. Teukolsky, Phys. Rev. D 57, 6181 (1998).

[16] P. Marronetti, G. J. Mathews, and J. R. Wilson, Phys. Rev. D 58, 107503 (1998).

[17] S. Bonazzola, E. Gourgoulhon, and J.-A. Marck, Phys. Rev. Lett. 82, 892 (1999).

[18] P. Marronetti, G. J. Mathews, and J. R. Wilson, Phys.
Rev. D 60, 087301 (1999).

[19] K. Uryū and Y. Eriguchi, Phys. Rev. D 61, 124023 (2000).

[20] K. Uryū, M. Shibata, and Y. Eriguchi, Phys. Rev. D 62, 104015 (2000).

[21] E. Gourgoulhon, P. Grandclément, and S. Bonazzola (2001), preprint gr-qc/0106015.

[22] P. Grandclément, E. Gourgoulhon, and S. Bonazzola (2001), preprint gr-qc/0106016.

[23] G. B. Cook (1994), unpublished notes.

[24] A. Lichnerowicz, J. Math. Pures et Appl. 23, 37 (1944).

[25] J. W. York, Jr., Phys. Rev. Lett. 26, 1656 (1971).

[26] J. W. York, Jr., Phys. Rev. Lett. 28, 1082 (1972).

[27] J. W. York, Jr., J. Math. Phys. 14, 456 (1973).

[28] J. M. Bowen, Gen. Relativ. Gravit. 11, 227 (1979).

[29] J. W. York, Jr., Phys. Rev. Lett. 82, 1350 (1999).

[30] J. R. Wilson and G. J. Mathews, in Frontiers in Numerical Relativity, edited by C. R. Evans, L. S. Finn, and D. W. Hobill (Cambridge University Press, Cambridge, England, 1989), pp. 306-314.

[31] S. Bonazzola, E. Gourgoulhon, and J.-A. Marck, Phys. Rev. D 56, 7740 (1997).

[32] G. B. Cook and J. W. York, Jr., Phys. Rev. D 41, 1077 (1990).

[33] J. Thornburg, Class. Quantum Gravit. 4, 1119 (1987).

[34] D. M. Eardley, Phys. Rev. D 57, 2299 (1998).

[35] L. Smarr and J. W. York, Jr., Phys. Rev. D 17, 1945 (1978).

[36] H. Pfeiffer (2001), private communication.

[37] E. Gourgoulhon and S. Bonazzola, Class. Quantum Gravit. 11, 443 (1994).

[38] A. Komar, Phys. Rev. 113, 934 (1959).

[39] R. Beig, Phys. Lett. 69A, 153 (1978).

[40] A. Ashtekar and A. Magnon-Ashtekar, J. Math. Phys. 20, 793 (1979).

[41] J. W. York, Jr., in Sources of Gravitational Radiation, edited by L. L. Smarr (Cambridge University Press, Cam- 
bridge, England, 1979), pp. 83-126.

[42] L. E. Kidder, M. A. Scheel, S. A. Teukolsky, E. D. Carlson, and G. B. Cook, Phys. Rev. D 62, 084032 (2000).

[43] J. P. Ostriker and J. E. Gunn, Astrophys. J. 157, 1395 (1969).

[44] J. B. Hartle, Astrophys. J. 161, 111 (1970).

[45] R. Rieth, in Mathematics of Gravitation. Part II. Gravitational Wave Detection, edited by A. Królak (Polish Academy of Sciences, Institute of Mathematics, Warsaw,
1997), pp. 71-74, proceedings of the Workshop on Mathematical Aspects of Theories of Gravitation held in Warsaw, February 29-March 30, 1996.

[46] A. Buonanno and T. Damour, Phys. Rev. D 59, 084006 (1999).

[47] H. Tagoshi, A. Ohashi, and B. J. Owen, Phys. Rev. D 63, 044006 (2001).

[48] K. Alvi, Phys. Rev. D 61, 124013 (2000). 